University of Nebraska - Lincoln

DigitalCommons@University of Nebraska - Lincoln

10-1999

\title{
Impact of Transmission Impairments on the Teletraffic Performance of Wavelength-Routed Optical Networks
}

\author{
Byrav Ramamurthy \\ University of Nebraska-Lincoln, bramamurthy2@unl.edu \\ Debasish Datta \\ IEEE \\ Helena Feng \\ IEEE \\ Jonathan P. Heritage \\ IEEE \\ Biswanath Mukherjee \\ IEEE
}

Follow this and additional works at: https://digitalcommons.unl.edu/csearticles

Part of the Computer Sciences Commons

Ramamurthy, Byrav; Datta, Debasish; Feng, Helena; Heritage, Jonathan P.; and Mukherjee, Biswanath, "Impact of Transmission Impairments on the Teletraffic Performance of Wavelength-Routed Optical Networks" (1999). CSE Journal Articles. 70.

https://digitalcommons.unl.edu/csearticles/70

This Article is brought to you for free and open access by the Computer Science and Engineering, Department of at DigitalCommons@University of Nebraska - Lincoln. It has been accepted for inclusion in CSE Journal Articles by an authorized administrator of DigitalCommons@University of Nebraska - Lincoln. 


\title{
Impact of Transmission Impairments on the Teletraffic Performance of Wavelength-Routed Optical Networks
}

\author{
Byrav Ramamurthy, Member, IEEE, Debasish Datta, Helena Feng, Student Member, IEEE, \\ Jonathan P. Heritage, Fellow, IEEE, Fellow, OSA, and Biswanath Mukherjee, Member, IEEE
}

\begin{abstract}
In a wavelength-routed optical network, a transmitted signal remains in the optical domain over the entire route (lightpath) assigned to it between its source and destination nodes. The optical signal may have to traverse a number of crossconnect switches (XCS's), fiber segments, and optical amplifiers, e.g., erbium-doped fiber amplifiers (EDFA's). Thus, while propagating through the network, the signal may degrade in quality as it encounters crosstalk at the XCS's and also picks up amplified spontaneous emission (ASE) noise at the EDFA's. Since these impairments continue to degrade the signal quality as it progresses toward its destination, the received bit error rate (BER) at the destination node might become unacceptably high. Previous work on the lightpath routing and wavelength assignment (RWA) problem assumed an ideal physical layer and ignored these transmission impairments. The main contribution of our work is to incorporate the role of the physical layer in setting up lightpaths by employing appropriate models of multiwavelength optical devices (XCS's and EDFA's) such that the BER of a candidate lightpath can be computed, in advance, to determine if this lightpath should be used for the call. Features from existing RWA algorithms are integrated with our on-line BER calculation mechanism. Our simulation studies indicate that employing BER-based call-admission algorithms has a significant impact on the performance of realistic networks.
\end{abstract}

Index Terms-Amplifier noise, bit error rate (BER), crosstalk, optical network, routing and wavelength assignment (RWA), transmission impairments, wavelength division multiplexing (WDM), wavelength routing.

\section{INTRODUCTION}

$\mathbf{I}$

$\mathrm{N}$ a wavelength-routed optical network, any transmitted signal remains in the optical domain over the entire route ${ }^{1}$

Manuscript received June 29, 1998; revised June 1, 1999. This work was supported in part by DARPA under Contracts DABT63-92-C-0031 and DAAH04-95-1-0487; the NSF under Grants NCR-9205755, NCR-9508239, and ECS-9521249; Pacific Bell; University of California MICRO Program; and a Lawrence Livermore National Laboratory Student Employeeship.

B. Ramamurthy is with the Department of Computer Science \& Engineering, University of Nebraska-Lincoln, Lincoln, NE 68588 USA.

D. Datta is with the Department of Electronics and Electrical Communications Engineering, Indian Institute of Technology, Kharagpur, West Bengal 721302 India.

H. Feng is with the Department of Applied Sciences, University of California, Davis, CA 95616 USA.

J. P. Heritage is with the Department of Electrical and Computer Engineering, University of California, Davis, CA 95616 USA.

B. Mukherjee is with the Department of Computer Science, University of California, Davis, CA 95616 USA (e-mail: mukherjee@cs.ucdavis.edu).

Publisher Item Identifier S 0733-8724(99)08011-1.

${ }^{1} \mathrm{~A}$ route is a path consisting of one or more fiber links, from the source to the destination. A route along with a chosen wavelength specifies the lightpath of the call. assigned to it between its source and destination nodes. We focus our attention on the class of optical networks wherein, in response to a given call request, a circuit-switched connection is established between the calling (source) and the called (destination) nodes on a single wavelength, provided a free wavelength is available over the desired lightpath, i.e., we do not consider wavelength conversion in this work. The teletraffic performance of such a network is generally evaluated in terms of the call blocking probability, which exhibits a strong dependence on the network topology, offered traffic pattern, number of wavelengths, and the routing and wavelength assignment (RWA) algorithm employed for establishing network connections.

In previous work on the (RWA) problem, e.g., [1]-[4], the network blocking has been estimated using analytical and simulation approaches under the assumption of an ideal physical layer that causes no impairment to a transmitted signal. Although an optical network, in general, offers improved transmission error characteristics compared to its copper or radio counterparts, we nevertheless need to consider these impairments and accommodate their impact in an optical network due to the following reasons.

In a wavelength-routed optical network spanning a large geographical area, an optical signal may traverse a number of intermediate nodes and long fiber segments. In order to enable the signal to propagate over the desired lightpath in the optical domain, a crossconnect switch (XCS) at each intermediate node employs passive and hence lossy switching, albeit through an active electro-optic control mechanism. The progressive losses incurred by the signal in all these nodes and long fiber segments necessitate the use of optical amplifiers [usually, erbium-doped fiber amplifiers (EDFA's)] at strategic locations in the network, possibly at each node and within the fiber segments [5]. Unfortunately, the XCS's and EDFA's-while offering transparent switching and loss compensation, respectively, for optical signals-may introduce significant transmission impairments, such as

- crosstalk generation when two or more optical signals copropagate through the same $\mathrm{XCS}^{2}$;

- generation of amplified spontaneous emission (ASE) noise in EDFA's while providing signal amplification;

\footnotetext{
${ }^{2}$ Among various forms of crosstalk, the most destructive one is homowavelength crosstalk, also known as in-band crosstalk [6].
} 
- saturation and wavelength dependence of EDFA gain, making the gain a traffic-dependent nondeterministic quantity.

The crosstalk and the ASE noise generated at every intermediate node copropagate along with the signal over the assigned lightpath; and all of them undergo variable gains at various wavelengths because of the traffic-dependent, nonflat gain spectra of EDFA's. Thus, a signal degrades in quality as it traverses through switches and fiber segments while propagating along its assigned lightpath toward its destination, and the signal-to-noise ratio (SNR) continues to decrease. When the signal finally arrives at the destination, the crosstalk and ASE noise that have accumulated along with the signal may result in significant degradation of the SNR, which might in turn increase the receiver bit error rate (BER) beyond its acceptable threshold. In order to examine the reliability of the physical layer, one therefore needs to capture all of these physical-layer limitations together and evaluate the achievable BER for a given lightpath.

This work is a novel attempt to evaluate network performance while taking into consideration the physical layer limitations. We try to capture the most significant impairments when we estimate the BER. Other impairments that are not treated here include: laser phase noise at the transmitter, fiber dispersion and nonlinearities [7], phase-to-intensity noise conversion caused by multiple reflections along the fiber path [8], [9], unbalanced gain for different optical channels [10] and effects of gain dynamics [11]-[13] originating from adding and droping optical signals at the EDFA's, and timing jitter at the receiver. These impairments can be significant especially when the bit rate is high and the network scale is large (national or international scale). Therefore our quasistatic model for estimating the BER gives a lower limit of the BER.

Since the BER on a lightpath would dynamically change with traffic variation (e.g., due to presence or absence of other copropagating lightpaths), it is useful to test the on-line BER for each lightpath that is considered for a call request. Thus, RWA algorithms which consider such BER constraints are more pragmatic, and they may lead to more efficient network operation.

The objective of the present work is to estimate the on-line $B E R$ on candidate routes and wavelengths before setting up a call. Note that the existence of other calls currently in progress, i.e., traffic variation, will affect the BER estimate (since they will affect the crosstalk in XCS's and the wavelength dependence and saturation of gains and ASE noise generation in EDFA's). One approach to call admission would be to set up a call on a lightpath with minimum BER. Another approach would be to establish a call on any lightpath with a BER lower than a certain threshold (e.g., $10^{-12}$ ); if no such lightpath is found, the call is blocked. Our work employs the second approach with a BER threshold (set to $10^{-12}$ in our numerical examples in this work). (Additional details of our approach, including the description of a simulator that we have developed, can be found in [14].)

In our approach, the BER of the candidate lightpath is computed during the admission phase of a call. Once the call has been set up in the network, its BER could vary slightly de-

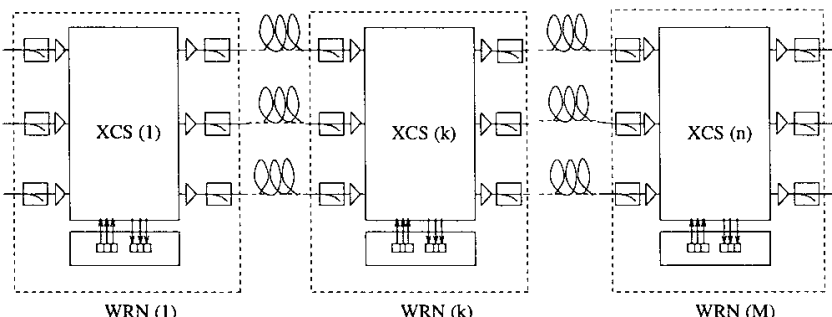

Fig. 1. Network components along a lightpath in a wavelength-routed optical network.

pending on the instantaneous traffic in the network-typically, the BER of an existing call in the network may increase slightly when a new call is established and it may decrease slightly when another ongoing call leaves the network. The RWA algorithm employed in this work ensures that a call is set up on a "good" route and wavelength when it is admitted into the network. The algorithm does not "shift" an existing call on to a different route or a different wavelength during its lifetime. This approach greatly simplifies the management of the network. Moreover, the calculation of the BER estimate of a call is highly computation-intensive and hence is undertaken only once per call. ${ }^{3}$ However, the approach described here can also be applied when the BER estimate of a call is required to be below a certain threshold throughout its duration in the network.

The rest of the paper is organized as follows. Section II describes the simulation procedure including the formalism to capture the system features both at the network and the physical layers. This section also includes the approaches adopted for enumeration of various crosstalk and ASE noise components along a lightpath and an analytical model to estimate the receiver BER on a candidate lightpath. Section III presents representative illustrative examples based on simulation experiments with on-line BER calculations, and discusses the salient features and observations from the experiments. Section IV concludes this study. Appendix A presents the analytical model used for EDFA gain computations and Appendix $\mathrm{B}$ describes the switch architecture used in this work.

\section{NetWORK SimUlation MODEL}

Below, we first describe the network architecture employed in this study. We then describe our novel hybrid simulation technique.

\section{A. Network Architecture}

Fig. 1 shows a portion of a wavelength-routed network traversed by a typical optical signal along a given lightpath. The lightpath consists of a number of intermediate wavelengthrouting nodes (WRN's, or simply, nodes) between the source and the destination nodes, interconnected by fiber segments.

\footnotetext{
${ }^{3}$ The simulation time for computing the BER estimates for the calls and generating the blocking probability at a particular load, for the sample mesh network with 15 nodes discussed later in this work, aggregated over 1 million call requests (on a lightly-loaded HP-UX 9000/778 machine) is around $2 \mathrm{~h}$ and $20 \mathrm{~min}$ which is equivalent to around $8.4 \mathrm{~ms}$ per call.
} 


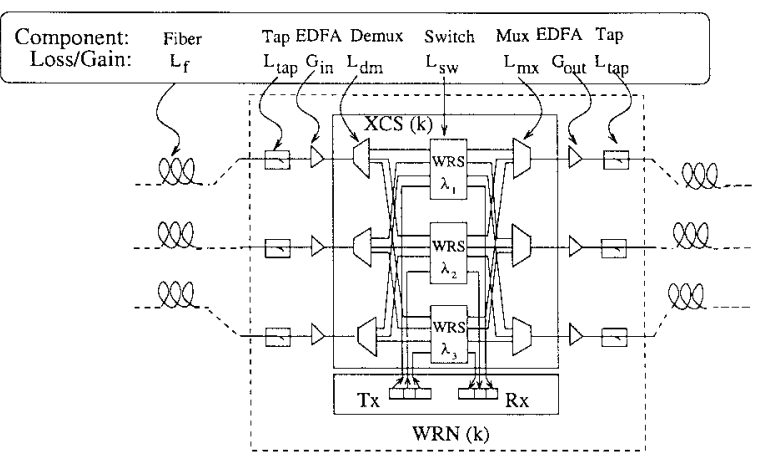

Fig. 2. Components and their loss/gain parameters in a wavelength-routing node $(\mathrm{WRN})$.

The constituent optical components in a given WRN include, in general, a crossconnect switch (XCS), a pair of EDFA's and optical power taps, on either side of the XCS at each port, for monitoring purposes. The EDFA on the input side (with small-signal gain, $G_{\text {in }}$ ) compensates exactly for the signal attenuation along the input fiber and the tap losses. The EDFA on the output side (with small-signal gain, $G_{\text {out }}$ ) compensates exactly for the losses at the crossconnect switch (XCS). The EDFA model used in this work is described in Appendix A. In this work, we assume that each WRN also contains a transmitter array (Tx) and a receiver array $(\mathrm{Rx})$, enabling local add/drop of any of the wavelengths at any of the nodes. The WRN's are connected through singlemode fibers which may employ in-line optical amplifiers for long-distance connectivity. Consistent with our neglect of fiber dispersion and nonlinearities, the fiber distances in our present study are not too long, and hence no in-line amplifiers are used. Note that this particular network architecture is provided only for illustration purposes; the approach presented in this work can be employed to study optical networks with different architectures and devices in a modular fashion. Below, we describe the architecture of the XCS, and its representative loss and crosstalk models used in this work.

Fig. 2 presents a block diagram for a possible realization of an XCS with three stages of components. The first stage consists of an array of demultiplexers; and it is followed by a stage of optical wavelength-routing switches (WRS) and a stage of multiplexers. All the demultiplexed signals on a given wavelength, say $\lambda_{1}$, are directed to the same optical switch $\left(\mathrm{WRS}-\lambda_{1}\right)$. The switch routes the signal toward the desired output port. Finally, the multiplexers combine the optical signals on all the wavelengths and pass them on to the desired output fiber. The number of optical switches in an XCS equals the number of incoming wavelengths, and each switch has at least $N$ input/output ports, where $N$ is the number of input/output fibers.

For the present work, we consider that the switch employs strictly nonblocking active splitter/active combiner architecture [15] based on titanium-diffused lithium niobate

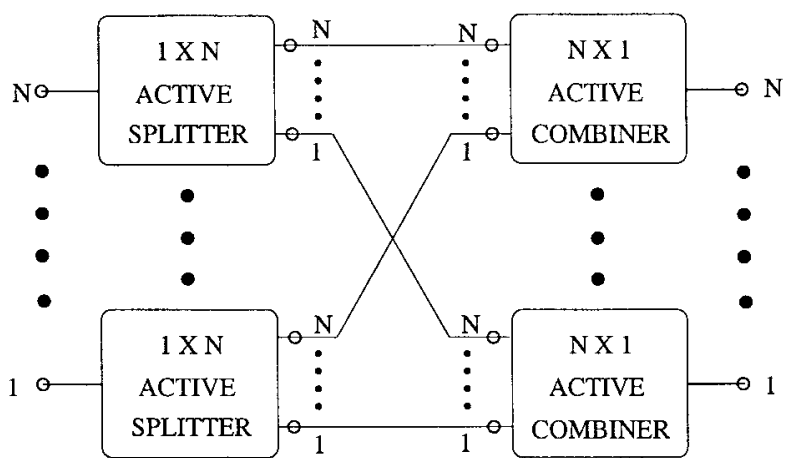

Fig. 3. Architecture of a $N \times N$ nonblocking space switch used in our model.

(Ti: $\mathrm{LiNbO}_{3}$ ) waveguides ${ }^{4}$. An $N \times N$ switch is constructed from $1 \times N$ active splitting elements and $N \times 1$ active combining elements, as shown in Fig. 3. This architecture requires $2 N(N-1)$ directional couplers, in which the basic building block is a $2 \times 2$ crossbar switch used as a $1 \times$ 2 or $2 \times 1$ element in the splitter and combiner stages, respectively. Signals can interfere with one another when they copropagate through the same switch, leading to crosstalk generation. Appendix B explains additional details of the switch architecture and crosstalk generation.

Note that the number of input (or output) ports in the switch is required to be a power of 2 . For example, the bidirectional ring network, which is used later in one of our illustrative examples, requires a $3 \times 3 \mathrm{XCS}$ at every $\mathrm{WRN}$, with two fiber ports on input and output sides along with local adddrop ports. The required switch geometry is realized by using a $4 \times 4$ switch, in which one of the four ports (usually the highest numbered one) on either side is unused.

The performance of such a network, in the presence of transmission impairments, is studied using a novel hybrid simulation technique, wherein we combine event-driven simulation of network-layer events with the on-line BER analysis of the physical-layer impairments. Below, we explain the hybrid approach using the block diagram in Fig. 4.

\section{B. Event-Driven Simulation Module}

Calls are generated between source-destination pairs in the network according to some specified distribution and the holding time of each call is also generated according to some distribution. For each call request, the event-driven simulation module begins as in a traditional manner, looking for a free wavelength on an available route. The route is chosen according to a predetermined method, e.g., shortestpath routing. If there is no route from the source to the destination or if no wavelengths are free along a chosen route, the call is blocked (i.e., dropped).

If a free wavelength is available, the lightpath is identified and the simulation is switched over to the on-line BER-evaluation module. For the call under consideration, the BER-evaluation module keeps track of the signal, noise, and crosstalk powers as the call progresses through the network.

\footnotetext{
${ }^{4}$ Note that other switch architectures can be accommodated, if so desired, by making appropriate adjustments to our switch model.
} 


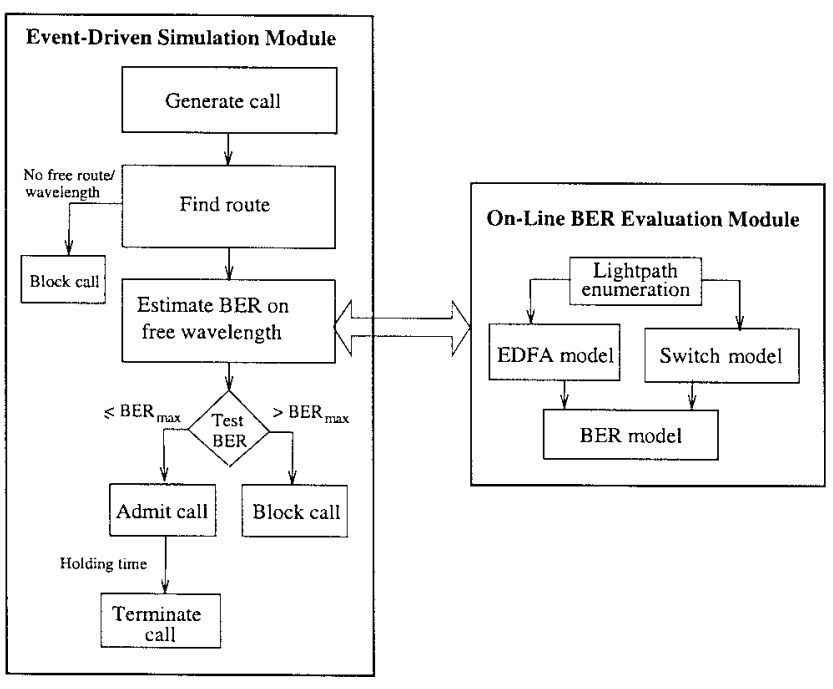

Fig. 4. Hybrid simulation technique.

The losses and gains in the network components traversed along the lightpath are computed, and the noise and crosstalk generated in the EDFA's and switches are enumerated. Finally, using the received signal, noise, and crosstalk powers at the destination, the BER model evaluates the receiver BER and sends back the BER estimate to the event-driven simulation module. Thereafter, a decision is made to set up or block the call depending on whether or not the BER estimate falls below some specified BER threshold.

If the BER estimate of the call exceeds its threshold, it is blocked. Otherwise, the call is admitted; and later, it is terminated upon its completion. This process is repeated for a large number of calls to the network. The blocking probability of the network $\left(P_{b}\right)$ is given by

$$
P_{b}=\frac{\text { Number of blocked calls }}{\text { Total number of offered calls }} .
$$

\section{On-Line BER Evaluation Module}

The computation of received power levels along the lightpath, during call admission, requires (1) the enumeration of all the events of signal, crosstalk, and ASE noise generation, and (2) their subsequent losses and gains at each node along the lightpath. Consider a lightpath which is to be established on wavelength $\lambda_{i}$ between nodes 1 and $M$ in a network as shown in Fig. 1. We express, at the output of the $k$ th intermediate node, the outbound powers of the signal $\left(p_{\mathrm{sig}}\left(k, \lambda_{i}\right)\right)$, crosstalk $\left(p_{x t}\left(k, \lambda_{i}\right)\right)$, and ASE noise $\left(p_{\text {ase }}\left(k, \lambda_{i}\right)\right)$ on wavelength $\lambda_{i}$, using the following recursive equations:

$$
\begin{aligned}
p_{\text {sig }}\left(k, \lambda_{i}\right)= & p_{\text {sig }}\left(k-1, \lambda_{i}\right) L_{f}(k-1, k) G_{\text {in }}\left(k, \lambda_{i}\right) \\
& \cdot L_{d m}(k) L_{s w}(k) L_{m x}(k) G_{\text {out }}\left(k, \lambda_{i}\right) L_{\text {tap }}^{2} \\
p_{x t}\left(k, \lambda_{i}\right)= & p_{x t}\left(k-1, \lambda_{i}\right) L_{f}(k-1, k) G_{\text {in }}\left(k, \lambda_{i}\right) \\
& \cdot L_{d m}(k) L_{s w}(k) L_{m x}(k) G_{\text {out }}\left(k, \lambda_{i}\right) L_{\text {tap }}^{2} \\
& +\sum_{j=1}^{J_{k}} X_{s w} p_{\text {in }}\left(j, k, \lambda_{i}\right) L_{s w}(k) L_{m x}(k) \\
& \cdot G_{\text {out }}\left(k, \lambda_{i}\right) L_{\text {tap }}
\end{aligned}
$$

$$
\begin{aligned}
p_{\text {ase }}\left(k, \lambda_{i}\right)= & p_{\text {ase }}\left(k-1, \lambda_{i}\right) L_{f}(k-1, k) G_{i n}\left(k, \lambda_{i}\right) \\
& \cdot L_{d m}(k) L_{s w}(k) L_{m x}(k) G_{\text {out }}\left(k, \lambda_{i}\right) L_{\text {tap }}^{2} \\
& +2 n_{s p}\left[G_{\text {in }}\left(k, \lambda_{i}\right)-1\right] h \nu_{i} B_{o} L_{d m}(k) \\
& \cdot L_{s w}(k) L_{m x}(k) G_{\text {out }}\left(k, \lambda_{i}\right) L_{\text {tap }} \\
& +2 n_{s p}\left[G_{\text {out }}\left(k, \lambda_{i}\right)-1\right] h \nu_{i} B_{o} L_{\text {tap }} .
\end{aligned}
$$

The loss and gain variables for various network components used above (generically, $L_{x}(k)$ for losses, and $G_{x}\left(k, \lambda_{i}\right)$ for gains) are indicated in Fig. 2. Further, $p_{i n}\left(j, k, \lambda_{i}\right)$ is the power of the $j$ th copropagating signal at the switch shared by the desired signal (i.e., the switch, WRS- $\lambda_{i}$, for wavelength $\lambda_{i}$ ) at the $k$ th node contributing to a first-order homowavelength crosstalk (switch crosstalk ratio $=X_{s w}$ ) with $J_{k}$ being the total number of such crosstalk sources at the $k$ th node (see Appendix B). $B_{o}$ is the optical filter bandwidth, $h$ is Planck's constant, $\nu_{i}$ is the optical frequency at $\lambda_{i}$, and $n_{s p}$ represents the spontaneous emission factor for the EDFA's. Note that the XCS's in a wavelength-routed network can generate two different types of crosstalk, viz., heterowavelength (interchannel) crosstalk in multiplexing and demultiplexing devices, and homowavelength (in-band) crosstalk in the space switches. In practice, the cumulative effect of homowavelength crosstalk causes the dominant impairment at the receiver as compared to its heterowavelength counterpart [16]. Therefore, we consider only the effect of homowavelength crosstalk in this work. The EDFA gains, $G_{\text {in }}\left(k, \lambda_{i}\right)$ and $G_{\text {out }}\left(k, \lambda_{i}\right)$, for each node at all the wavelengths are evaluated using a simplified model (see Appendix A), similar to [17], which takes into account the major physical phenomena in EDFA's, such as multiwavelength signal propagation, and self-saturation and cross-saturation of the EDFA gains by the traffic-dependent signal channels.

Having completed the enumeration process as above till the $M$ th node, the BER-evaluation module computes the powers of the composite electrical noise for binary zero and one receptions, which include the receiver thermal and shot noise components and the electrical noise components resulting from the signal-crosstalk and signal-ASE beats. The composite electrical noise powers and the received photocurrent are then used to evaluate the BER at the receiver, as described below.

\section{BER Model}

The lightwave received at the destination node in the presence of crosstalk and ASE contributions can be expressed as:

$$
E_{R}(t)=A \cos \left(2 \pi \nu_{i} t+\phi(t)\right)+E_{x t}(t)+E_{\text {ase }}(t)
$$

The first term in (5) represents the lightwave for the desired signal component at frequency $\nu_{i}$, with $A$ and $\phi(t)$ as the amplitude and the phase noise of the signal while the second and the third components represent the lightwaves corresponding to the accumulated crosstalk and ASE noise components, respectively. The received lightwave, after photodetection, produces a photocurrent given by

$$
i_{p}(t)=R_{\lambda}\left\langle E_{R}^{2}(t)\right\rangle+i_{s h}(t)+i_{\mathrm{th}}(t)
$$

where the first term represents the square-and-average response of the photodetector to the incident lightwave $E_{R}(t)$ 
with $R_{\lambda}$ representing the responsivity of the photodetector, the second term is the shot noise produced by the incident lightwave, and the third term accounts for the receiver thermal noise. The first term of $i_{p}(t)$ in (5) can be expressed as

$$
\begin{aligned}
R_{\lambda}\left\langle E_{R}^{2}(t)\right\rangle= & i_{s}(t)+i_{s x}(t)+i_{s s p}(t) \\
& +i_{x x}(t)+i_{s p s p}(t)+i_{x s p}(t)
\end{aligned}
$$

where $i_{s}(t)$ represents the desired signal component while the remacining terms account for the beat noise components between signal and crosstalk $\left(i_{s x}(t)\right)$, signal and $\operatorname{ASE}\left(i_{s s p}(t)\right)$, crosstalk and itself $\left(i_{x x}(t)\right)$, ASE and itself $\left(i_{s p s p}(t)\right)$, and crosstalk and ASE $\left(i_{x s p}(t)\right)$. Considering that the dominant beat noise terms are contributed by the signal-crosstalk and the signal-ASE combinations, and representing all the noise components as a combined noise process $n_{i}(t)$, we approximate (7) as

$$
\begin{aligned}
i_{p}(t) & =i_{s}(t)+\left[i_{s x}(t)+i_{s s p}(t)+i_{s h}(t)+i_{\mathrm{th}}(t)\right] \\
& =I_{s i}+n_{i}(t) \\
& =R_{\lambda} s\left(N, \lambda_{i}\right) b_{i}+n_{i}(t)
\end{aligned}
$$

where $i$ in the subscripts of all the terms in (9) and (10) represents the data bit ( 1 or 0 ) being received, $I_{s i}$ with $i=1$ or 0 represents the corresponding signal components of the photocurrent, $s\left(N, \lambda_{i}\right)$ represents the average value of the received optical signal power, and $b_{i}=2$ or 0 for $i=1$ or 0 , respectively. The combined electrical noise $n_{i}(t)$ is modeled as a zero-mean Gaussian random process with a variance given by

$$
\sigma_{i}^{2}=\sigma_{s x i}^{2}+\sigma_{s s p i}^{2}+\sigma_{s h i}^{2}+\sigma_{\mathrm{th}}^{2}
$$

where the corresponding noise variances are given by

$$
\begin{aligned}
\sigma_{s x i}^{2}= & 2 \xi_{\mathrm{pol}} R_{\lambda}^{2} b_{i} s\left(N, \lambda_{i}\right) p_{x t}\left(N, \lambda_{i}\right) \\
\sigma_{s s p i}^{2}= & 4 R_{\lambda}^{2} b_{i} s\left(N, \lambda_{i}\right) p_{\text {ase }}\left(N, \lambda_{i}\right) B_{e} / B_{\circ} \\
\sigma_{s h i}^{2}= & 2 q R_{\lambda}\left(b_{i} s\left(N, \lambda_{i}\right)\right. \\
& \left.+p_{x t}\left(N, \lambda_{i}\right)+p_{\text {ase }}\left(N, \lambda_{i}\right)\right) B_{e} \\
\sigma_{\text {th }}^{2}= & \eta_{\text {th }} B_{e} .
\end{aligned}
$$

In (12)-(14), $s\left(N, \lambda_{i}\right), p_{x t}\left(N, \lambda_{i}\right)$, and $p_{\text {ase }}\left(N, \lambda_{i}\right)$ are as defined in (2)-(4). Equation (12) gives the variance of signalcrosstalk beat noise with $\xi_{\mathrm{pol}}(=1 / 2$, see [18]) representing the polarization mismatch factor between the signal and crosstalk lightwaves. Equation (13) provides the variance of signal-ASE beat noise with $B_{\circ}$ and $B_{e}$ representing the optical and the electrical bandwidths of the receiver. Equation (15) gives the variance of thermal noise with $\eta_{\text {th }}$ representing the spectral density of the thermal noise current in the optical receiver.

\begin{tabular}{|c|c|}
\hline Parameter & Value \\
\hline Number of wavelengths & 8 \\
\hline Wavelength-spacing & $100 \mathrm{GHz}$ \\
\hline Wavelengths (in nn) & $\begin{array}{l}(1546.99,1547.80,1548.60,1549.40 \\
1550.20,1551.00,1551.80,1552.60)\end{array}$ \\
\hline Bit rate per channel $(r)$ & $1 \mathrm{Gbps}$ \\
\hline Electronic bandwidth $\left(B_{e}\right)$ & $0.7 r$ \\
\hline Multiplexer loss $\left(L_{m x}\right)$ & $4 \mathrm{~dB}$ \\
\hline Demultiplexer loss $\left(L_{d t r}\right)$ & $4 \mathrm{~dB}$ \\
\hline Switch element insertion loss $\left(L_{s}\right)$ & $1 \mathrm{~dB}$ \\
\hline Waveguide/fiber coupling loss $\left(L_{w}\right)$ & $1 \mathrm{~dB}$ \\
\hline Switch loss $\left(L_{s w}\right)$ (from [15]) & $2 \log _{2} N L_{s}+4 L_{w}$ dB (for a $\mathrm{N} \times \mathrm{N}$ switch) \\
\hline Tap loss $\left(L_{\text {tap }}\right\}$ & $1 \mathrm{~dB}$ \\
\hline Fiber loss $\left(L_{f}\right)$ & $0.2 \mathrm{~dB} / \mathrm{km}$ \\
\hline Input EDFA Gain $\left(G_{i n}\right)$ & $22 \mathrm{~dB}$ \\
\hline Output EDFA Gain $\left(G_{\text {out }}\right)$ & $\begin{array}{l}16 \mathrm{~dB} \text { (for sample ring network); } \\
18 \mathrm{~dB} \text { at nodes } 1,6,7,13 \text {, and } \\
16 \mathrm{~dB} \text { elsewhere (for sample mesh network) }\end{array}$ \\
\hline ASE factor $\left(n_{s p}\right)$ & 1.5 \\
\hline RMS thermal current $/ \sqrt{\text { bandwidth }}\left(\sqrt{\eta_{t h}}\right)$ & $5.3 \times 10^{-12} \mathrm{Amp} / \sqrt{\mathrm{Hz}}$ \\
\hline Max. Iaser power $\left(P_{t}\right)$ & $0 \mathrm{dBm}(1 \mathrm{~mW})$ \\
\hline Switch crosstalk ratio $\left(X_{s w}\right)$ & $25 \mathrm{~dB}, 30 \mathrm{~dB}$ \\
\hline Excess small-signal gain $(d G)$ & $0 \mathrm{~dB}, 3 \mathrm{~dB}$ \\
\hline
\end{tabular}

The receiver BER is evaluated with a given decision threshold choice, $I_{\mathrm{TH}}$. One can minimize the BER by an optimum selection of $I_{\mathrm{TH}}$. The optimum selection of $I_{\mathrm{TH}}$ can be effected by using the following expression:

$$
I_{\mathrm{TH}}=\frac{\sigma_{0} I_{s 1}}{\sigma_{0}+\sigma_{1}}
$$

However, an optimum choice of $I_{\mathrm{TH}}$ from (16) can only be made with a prior knowledge of the received power levels of
TABLE I

System Parameters and Their Values used in the Models

signal, crosstalk, and ASE components. In the present network architecture, since all of these powers are dependent on the assigned lightpath and hence variable, one cannot optimize the threshold in a static sense. On the other hand, dynamic control of threshold for each lightpath would need a significant communication overhead for the network. In view of the above and assuming a perfect laser extinction (i.e., $b_{0}=0$, and hence $I_{s 0}=0$ ), we fix the receiver threshold $I_{\mathrm{TH}}$ at $I_{s 1} / 2$.

Using the above threshold and noise variances, we express the receiver BER as

$$
P_{b}=0.25\left[\operatorname{erfc}\left(\frac{I_{s 1}-I_{\mathrm{TH}}}{\sqrt{2} \sigma_{1}}\right)+\operatorname{erfc}\left(\frac{I_{\mathrm{TH}}}{\sqrt{2} \sigma_{0}}\right)\right] .
$$

The above expression for BER assumes negiligible timing jitter in the recovered clock of the receiver. In reality, the recovered clock may have timing jitter introduced by the inherent pattern randomness of the received binary sequence and the receiver noise components. However, an efficient twolevel line coding technique (such as, $\mathrm{mBnB}$ codes [19]) is usually employed in optical transmitters to introduce frequent data transitions in the bit stream which in turn reduces the clock timing jitter significantly in the receiver; the present BER model handles such optical receivers, wherein we ignore the impact of the small residual timing jitter in the recovered clock.

\section{ILLUSTRATIVE NUMERICAL EXAMPLES AND DisCUSSION}

In this section, we present for illustration purposes some representative numerical examples of our simulation experiments employing the proposed models of the physical-layer phenomena and their impact on the blocking performance of networks. In Table I, we present the system/device parameters used in these experiments. 


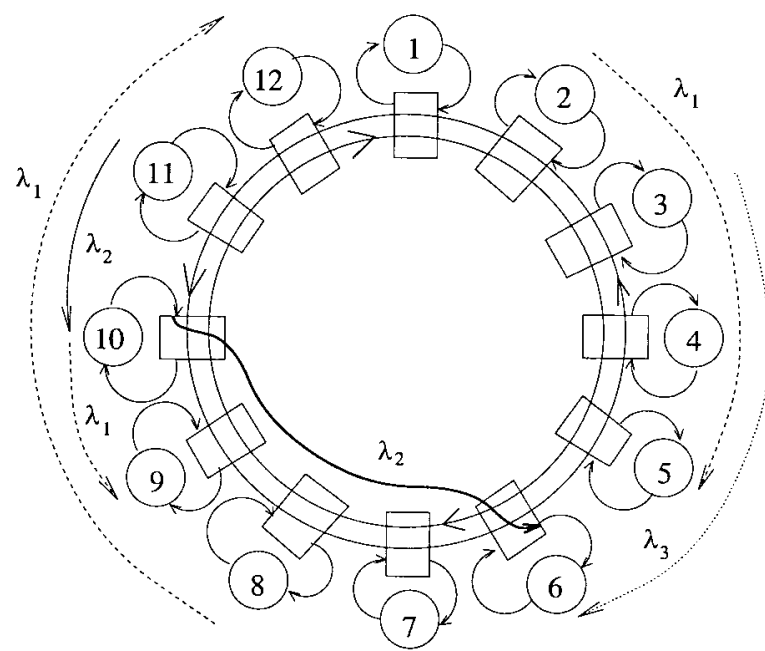

Fig. 5. Calls at the instant of our simulation snapshot in a bidirectional ring network.

First, we apply our on-line BER-based call admission approach to a bidirectional ring network, shown in Fig. 5, with 12 nodes and an internode distance of $100 \mathrm{~km}$. We employ shortest-path routing of lightpaths.

We consider a tagged call that is set up from node 10 to node 6 on wavelength $\lambda_{2}$ through the intermediate nodes 9 , 8 , and 7. At this instant of our simulation snapshot, the other ongoing calls are from node 2 to node 5 , from node 8 to node 12 , and from node 10 to node 9 on wavelength $\lambda_{1}$; from node 11 to node 10 on wavelength $\lambda_{2}$; and from node 3 to node 6 on wavelength $\lambda_{3}$. Fig. 6 shows the powers of the received signal, ASE noise, and crosstalk at the destination node (4) and at the intermediate nodes. Note that the signal power drops, as the call propagates through the network. Recall that the smallsignal gain of each amplifier in the network was set to be exactly equal to the losses at intervening network components. Hence, any reduction in available gain due to amplifier gain saturation results in inadequate compensation for the signal loss. Also, the crosstalk for this tagged call follows a similar profile as the signal due to the absence of any fresh crosstalk en route. ${ }^{5}$ However, the ASE noise grows due to accumulation of ASE at each EDFA stage. The resulting BER's of the tagged call at the receivers in nodes $9,8,7$, and 6 turn out to be $10^{-25}, 10^{-24}, 10^{-22}$, and $10^{-20}$, respectively. We note that the BER of the tagged call grows as it traverses more and more links because of the degradation in its signal-to-noise ratio (SNR).

Next, we consider the mesh network shown in Fig. 7. This network consists of 15 nodes with an internode distance of $100 \mathrm{~km}$. The mesh network is formed as a "set of interconnected rings" as is typical of telecommunication networks. We measure the dynamic performance (see Fig. 8) of the mesh network without BER constraints (the "ideal" case) and with BER constraints under several conditions (the "realistic" cases). We assume the following: a) Poisson

\footnotetext{
${ }^{5}$ The occurrence of crosstalk requires the presence of interfering calls in the network which share the same switch with the tagged call in a manner described in Appendix B.
}

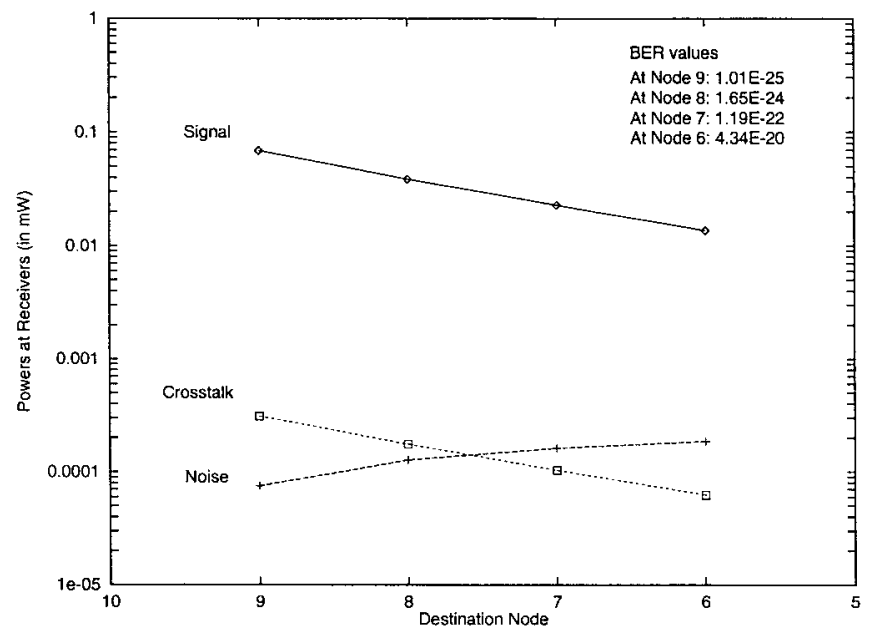

Fig. 6. Progress of a tagged call from Node 10 to Node 6 in the bidirectional ring network. The figure shows the signal, noise, and crosstalk powers and the BER values at the receivers of the intermediate nodes $(9,8$, and 7$)$ and the destination node (6) on wavelength $\lambda_{2}$ for this call.

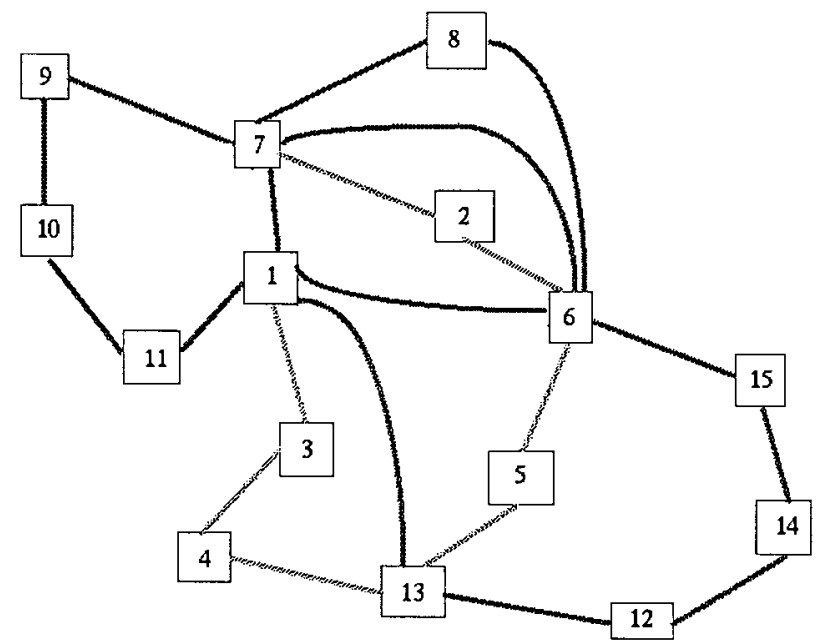

Fig. 7. A mesh network.

call arrivals, b) exponential call holding time, c) uniform distribution of source-destination pair for each call, d) shortestpath routing of lightpaths, and e) loss of blocked calls. The BER threshold (for the realistic cases) was set to $10^{-12}$, and one million call requests were simulated. We employ two standard wavelength-assignment algorithms: 1) the First-Fit algorithm where the first available wavelength in a predetermined order is used to set up a call and 2) the Random algorithm where a wavelength is chosen at random from among the free wavelengths on the route. For the realistic cases, in order to study the impact of switch crosstalk, we vary the parameter $X_{s w}$ (switch crosstalk ratio, see Appendix B). Further, in order to study the impact of gain saturation, we vary the parameter $d G$, which denotes the excess smallsignal gain, in $\mathrm{dB}$, at each amplifier in the network. This small-signal gain is added to ensure that enough gain is supplied to a signal even though the amplifier gain may be saturated. 


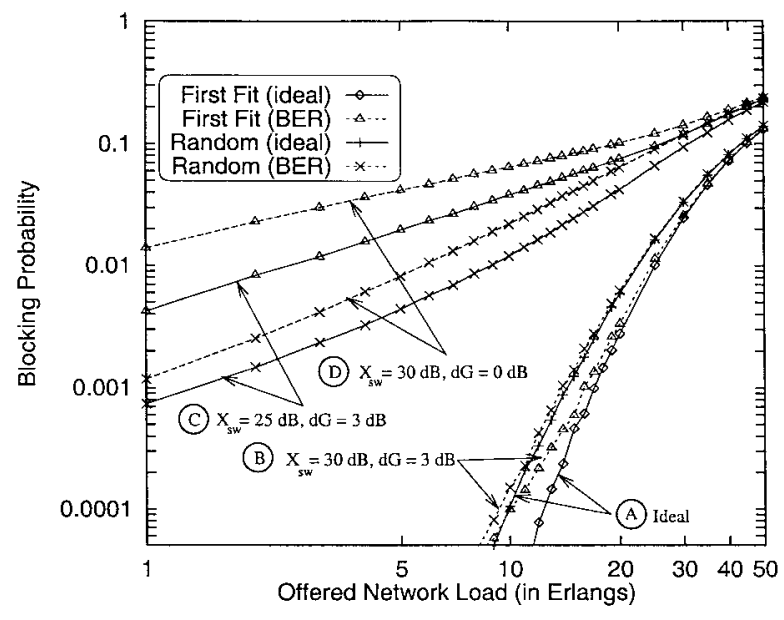

Fig. 8. Blocking probability versus load for the mesh network.

From the results shown in Fig. 8, we observe the following.

- For both wavelength-assignment algorithms (First-Fit and Random), the blocking performance for the realistic case with $X_{s w}=30 \mathrm{~dB}$ and $d G=3 \mathrm{~dB}$ is quite similar to that for the ideal case (see curves marked A and B in Fig. 8).

- When the switch crosstalk is increased to $X_{s w}=25$ $\mathrm{dB}$ (see curves marked $\mathrm{C}$ in Fig. 8), or when there is inadequate excess small-signal gain to compensate for saturation, i.e., $d G=0 \mathrm{~dB}$ (see curves marked $\mathrm{D}$ in Fig. 8), blocking in the network increases because of increased BER owing to reduced SNR. The importance of saturation is confirmed when the experiments corresponding to curves marked D in Fig. 8 are repeated with gain saturation turned off. The resulting curves are indistinguishable from those for the ideal case (i.e., the curves marked $\mathrm{A}$ in Fig. 8). We also note that, although the blocking probabilities with BER constraints are generally higher compared to those for the ideal case (especially so for light loads), they offer a more realistic performance measure of the network.

- The First-Fit wavelength assignment algorithm performs better (lower blocking) than the Random algorithm for the ideal case, as expected (see curves marked $\mathrm{A}$ in Fig. 8). However, when we incorporate BER constraints in a network with poor crosstalk or inadequate gain compensation (curves marked C or D in Fig. 8), we notice that the Random algorithm actually performs better than First-Fit! This is explained by the fact that the Random algorithm results in less homowavelength crosstalk in the network because calls are distributed more evenly over all the available wavelengths, as opposed to First-Fit, which tends to reuse ("pack") wavelengths, leading to greater interference between signals at the switches.

\section{CONCLUSION}

This work investigated the impact of transmission impairments on blocking performance of wavelength-routed optical networks. The transmission impairments of physical layer include crosstalk in the wavelength-routing nodes, wavelength dependence and saturation of amplifier gains and ASE generation in EDFA's, along with the receiver's shot as well as thermal noise components. The impact of these impairments was taken into consideration in the wavelength-assignment algorithm using a novel hybrid simulation technique. The technique combined event-driven simulation of network-layer events with on-line evaluation of BER along the lightpath, while incorporating the nondeterministic traffic-dependent EDFA gains and switch crosstalks. The study indicates that employing BER-based call-admission algorithms has a significant impact on the performance of realistic wavelength-routed optical networks.

\section{APPENDIX A EDFA MODEL}

An ideal optical amplifier would provide fixed gain for all wavelengths (channels), be noise-free, and introduce no interchannel crosstalk. All physically-realizable amplifiers depart from the ideal for fundamental physical reasons, and the EDFA is no exception. From the point of view of steady-state network performance, the three most significant departures from perfection that must be incorporated in a realistic EDFA model are i) gain dispersion, ii) gain saturation, and iii) noise. Gain dispersion refers to wavelength-dependent gain, while gain saturation is the signal-power-dependent gain compression that an amplifiers suffers when the input signal strength becomes large. Finally, an optical amplifier adds significant noise to the signal channel owing to unavoidable spontaneous emission. Other amplifier imperfections such as cross-channel modulation and refractive index dispersion can become important in long-distance links.

Our interest in exploring the impact of transmission impairments on blocking performance of optical networks requires that the optical amplifier model faithfully reproduce the most significant departures from the ideal. We have implemented a class of amplifier models that are capable of approximating, to a reasonable degree, the power gain performance of an EDFA line amplifier including gain dispersion, gain saturation, and ASE noise power. We do not attempt to calculate optical field propagation as that would require extensive computation time.

EDFA's have been extensively investigated experimentally and theoretically during the last decade. Thanks to the nature of the spectroscopy of the Erbium ion embedded in a random glass environment, a first principle analysis of EDFA gain is complicated and a complete analysis of gain dynamics including inhomogeneous broadening has not been presented. Nonetheless, Desurvire and Giles [17], [10] have developed models capable of describing gain dispersion and wavelength-dependent saturation. Simplified models, such as those of Hodgkinson [20] and Saleh [21] approximate amplifier behavior, including steady-state gain saturation with reasonable computation time. We include in our model the most essential aspects of the physics of the EDFA, such as multiwavelength signal propagation, selfsaturation and cross-saturation of the gain by the various signal channels as well as added ASE noise in much the same way as in [10]. 
The basic equations that describe the growth of $N$ signals propagating along the amplifier are

$$
\frac{d p_{i}}{d z}=\alpha\left(\nu_{i}, p_{1}, \ldots p_{N}\right) \cdot p_{i}
$$

where $i$ is the signal identifier, i.e., $i=1,2, \ldots, N ; p_{i}$ is the power of the $i$ th signal; $\nu_{i}$ is the frequency; $\alpha$ is the gain coefficient. A fourth-order Runge-Kutta method with adaptive stepsize control is implemented to integrate the set of coupled ordinary differential equations. EDFA gain curves are modeled by a fitting set of $M$ Lorentzian lines with shifted centers, and adjustable line width and line strength (Reasonable fits are obtained for $M=7$ lines.). Each Lorentzian is allowed to saturate by the influence of the signal powers with saturation strength adjusted according to each signal detuning. That is

$$
\begin{aligned}
\alpha(\nu) & =\sum_{j=1}^{M} \alpha_{j}\left(\nu, p_{1}, \ldots p_{N}\right) \\
\alpha_{j}\left(\nu, p_{1}, \ldots, p_{N}\right) & =\frac{\alpha_{j}^{0}}{1+y_{j}(\nu)^{2}} \cdot \frac{1}{1+\frac{1}{1+y_{j}(\nu)^{2}} \sum_{i=1}^{N} \frac{p_{i}}{p_{j}^{\text {sat }}}} \\
\alpha_{j}\left(\nu, p_{1}, \ldots, p_{N}\right) & =\frac{\alpha_{j}^{0}}{1+y_{j}(\nu)^{2}} \cdot \frac{1}{1+\frac{1}{1+y_{j}(\nu)^{2}} \sum_{i=1}^{N} \frac{p_{i}}{p_{j}^{\text {sat }}}}
\end{aligned}
$$

where $\nu_{j}^{0}$ is the central frequency of the $j$ th Lorentzian; $\alpha_{j}^{0}$ is the small-signal gain at line center, $\Delta \nu_{j}$ is the line width; $p_{j}^{\text {sat }}$ is the saturation power at line center; and $y_{j}(\nu)$ is the detuning with respect to the $j$ th line center. One set of parameters is chosen such that the small-signal gain and gain saturation behavior closely approximate published experimental data ([10, p. 339]). In our model, the ASE is assumed to be small as compared to the signal so that it does not participate in gain saturation. ASE noise power associated with the $i$ th signal within an optical bandwidth $B_{0}$ is

$$
p_{\text {ase(self) }}\left(\lambda_{i}\right)=n_{s p}\left(G\left(\lambda_{i}\right)-1\right) h \nu_{i} B_{o}
$$

where $n_{s p}>1$ is the ASE factor ([10, p. 77]), $h$ is the Planck's constant, $G\left(\lambda_{i}\right)$ is the saturated gain at wavelength $\lambda_{i}=c / \nu_{i}$ with $c$ as the velocity of light. The total output ASE noise at $\lambda_{i}$ in a bandwidth of $B_{\circ}$ is

$$
p_{\text {ase }}\left(\lambda_{i}\right)=p_{\text {ase(self) }}\left(\lambda_{i}\right)+p_{\text {ase(in) }}\left(\lambda_{i}\right) G\left(\lambda_{i}\right)
$$

where $p_{\text {ase(in) }}\left(\lambda_{i}\right)$ represents the input noise power at the same waveband.

We consider only forward propagation of signals, noise, and crosstalk in steady state, and since the current network model is a steady-state model, we do not consider EDFA gain dynamics. As the above equations indicate, our amplifier model is based on a best qualitative fit to a measured EDFA line shape but is not based on detailed underlying spectroscopy. Nonetheless,

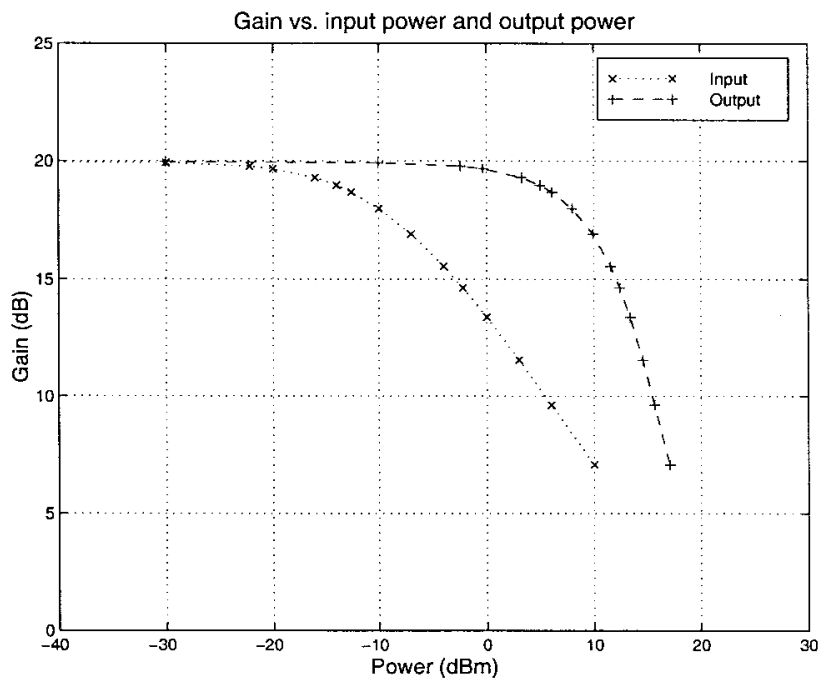

Fig. 9. Calculated gain saturation curves for input and output power variation.

the small-signal fit is excellent and the saturated gain spectra may be fit to individual amplifiers by allowing for adjustable saturation parameters. For purposes of computational efficiency, the relatively-flat gain spectrum in the neighborhood of $1550 \mathrm{~nm}$ is approximated in this initial working model with a single $(M=1)$ broad Lorentzian linewidth of $40 \mathrm{~nm}$, i.e., we take only one term from the sum in the expression [see (19)] for the gain. This choice of a relatively broad flat linewidth, and our choice of $100 \mathrm{GHz}$ channel spacing combine to make our network study not strongly sensitive to gain dispersion.

We have tested our model by choosing parameters such that our model reproduces the single channel saturation behavior of a measured EDFA amplifier ([10, p. 339]). This amplifier has a small-signal gain of $G_{\max }=33.6 \mathrm{~dB}$ and length $L=43.0 \mathrm{~m}$. We calculate $P_{\text {sat }}=13.7 \mathrm{~mW}(11.4 \mathrm{dBm})$ from the measured amplifier input saturation power. The network study presented here dictated the choice of three different amplifiers with small signal gains of 16,18 , and $22 \mathrm{~dB}$. These design criteria are met in our amplifier model by using the parameters for the $43.0 \mathrm{~m}$ amplifier described above, but by reducing the lengths accordingly. The calculated input power and output power amplifier saturation curves for our hypothetical amplifier are shown in Fig. 9. Further amplifier realism will be injected into our simulations through the course of our studies of BER-based call blocking.

\section{APPENDIX B \\ SWITCH MODEL}

Consider the architecture of a $N \times N$ switch shown in Fig. 3. This switch architecture provides strictly nonblocking, ${ }^{6}$ point-to-point connectivity. The switch consists of a stage of $1 \times N$ active splitters followed by a stage of $N \times 1$ active combiners [15]. The active splitter portion of the $N \times N$ switch consists of $\log _{2} N$ stages of $1 \times 2$ switch elements; the active combiner portion consists of $\log _{2} N$ stages of $2 \times 1$ switch

\footnotetext{
${ }^{6}$ The switch architecture is considered nonblocking because any input can always be connected to any unused output.
} 


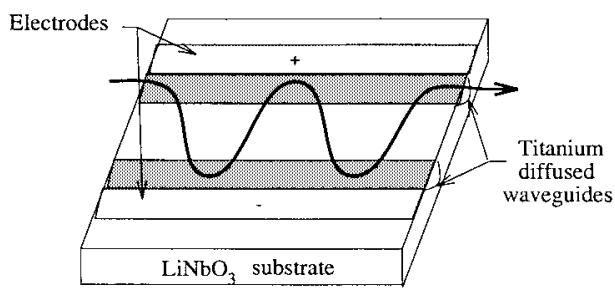

Fig. 10. Illustration of a directional coupler in the bar state.

elements. A total of $2 N(N-1)$ switch elements is employed in a $N \times N$ switch. Both the $1 \times 2$ and the $2 \times 1$ switch elements employ a directional coupler (conceptually, a $2 \times 2$ photonic switch) as the basic building block.

The directional couplers studied in this work are titaniumdiffused lithium niobate $\left(\mathrm{Ti}: \mathrm{LiNbO}_{3}\right)$ based devices (see Fig. 10, from [22]). Two optical (titanium) waveguides are diffused into a lithium niobate substrate and are brought close together to allow the energy from one waveguide to couple to the other. Electrodes are placed over the waveguides in the region where coupling occurs. By default, the directional couplers are set in a cross state with no voltage applied. To force the directional coupler to go from the cross state to the bar state, an electric field is applied through the electrodes [22]. Each of the $1 \times N$ splitters and $N \times 1$ combiners is manufactured on its own individual $\mathrm{LiNbO}_{3}$ substrate. Partitioning the architecture over several substrates allows for large switch dimensions without complex integration on individual substrates.

The insertion loss for the switch architecture is dependent upon the number of switch elements (i.e., directional couplers) that a signal must traverse. Note that all signals in a given $N \times N$ switch travel through the same number (viz., $2 \log _{2} N$ ) of individual switch elements in this architecture. Each switch element has a characteristic loss, $L_{s}$, in $\mathrm{dB}$, associated with it. This term $\left(L_{s}\right)$ accounts for material absorption and scattering losses incurred as the signal propagates through a given length of $\mathrm{LiNbO}_{3}$ waveguide and for losses due to incomplete coupling within the directional coupler. An additional attenuation occurs during the transfer of the signal on and off of the $\mathrm{LiNbO}_{3}$ substrate. This waveguide/fiber coupling loss is represented by $L_{w}$, and includes the Fresnel reflection losses and mode mismatch losses. Coupling between waveguide and fiber occurs at four locations along the path of a signal at any $N \times N$ switch, viz. at the input and output of both the splitter and combiner stages (denoted by the empty circles in Fig. 3). Thus, the insertion loss of the $N \times N$ switch in Fig. 3 is given by $2 \log _{2} N L_{s}+4 L_{w} \mathrm{~dB}$, where $L_{s}$ is the switch element insertion loss and $L_{w}$ is the waveguide/fiber coupling loss [15].

Crosstalk occurs in a switch when a portion of a signal "leaks" into another signal as they copropagate through the switch fabric. The ratio of the power at the unselected output port over the total input power in a switch element is referred to as the crosstalk ratio of the switch element $(X)$. Consider the $2 \times 2$ switch shown in Fig. 11. Suppose a call has been set up in the switch between input port 2 and output port 1 . Now we would like to establish a new call between input port 1 and

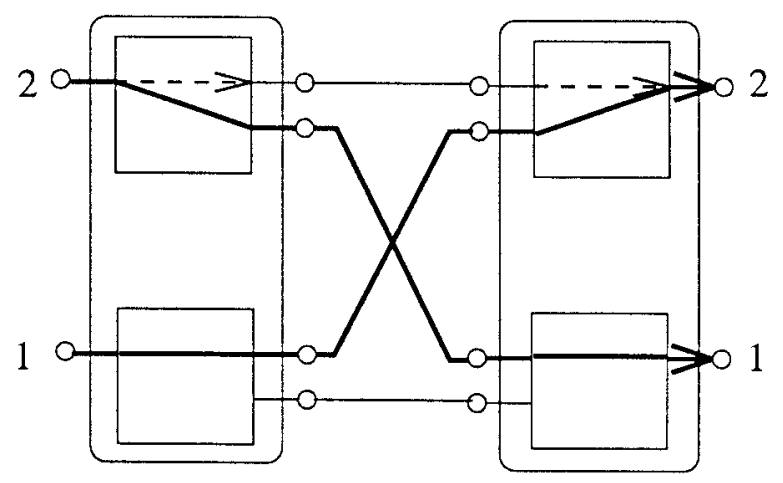

Fig. 11. Crosstalk in a $2 \times 2$ switch.

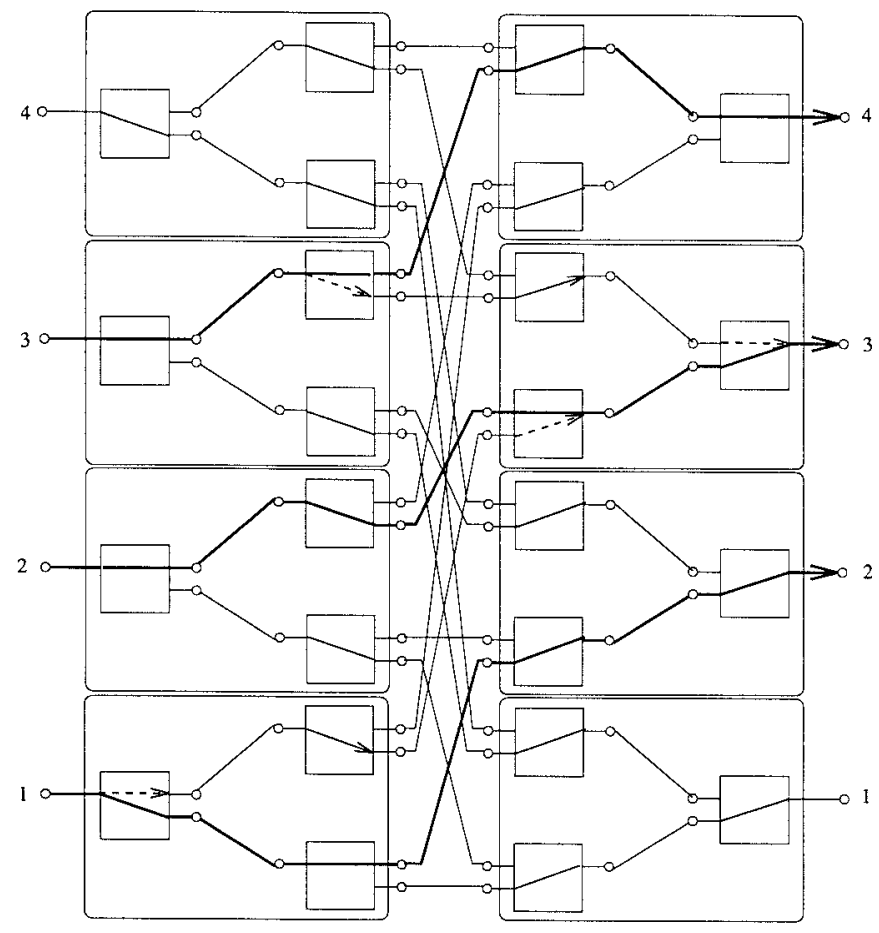

Fig. 12. Crosstalk in a $4 \times 4$ switch. Existing calls from input port 3 to output port 4 and from input port 1 to output port 2 both interfere with the new call from input port 2 to output port 3 .

output port 2. We find that a portion of the optical power on the existing call mixes with the new call at the combiner stage (depicted as dashed lines in Fig. 11). In this architecture, we find that crosstalk must occur in a single splitter element and in a single combiner element in order for an existing signal to interfere with the new signal. This is referred to as the first-order crosstalk effect in this architecture and results in a fraction, $X_{s w}=X^{2}$, of the interfering signal mixing with the new signal. The first-order switch crosstalk is the square of the crosstalk ratio of the individual element since the interfering signal leaks into the unselected port at two intermediate switch elements before it encounters the new signal. Fig. 12 shows the occurrences of crosstalk in a $4 \times 4$ switch with two calls (the first, from input port 3 to output port 4 and the second, from input port 1 to output port 2) already established. Note that the new call (from input port 2 to output port 3 ) is affected by both of these existing calls through crosstalks at different switch 
elements along its path within the switch. An algorithm that determines whether a given call (from input port $i_{1}$ to output port $o_{1}$ ) interferes with another call (from input port $i_{2} \neq i_{1}$ to output port $\left.o_{2} \neq o_{1}\right)$ in a $N \times N$ switch is described in [14]. Higher order crosstalk terms $\left(X^{3}, X^{4}\right.$, etc.) are possible in large switches; however, their effects are negligible compared to the first-order terms, and hence, they are not considered in this work.

\section{REFERENCES}

[1] K. Bala, T. E. Stern, D. Simchi-Levi, and K. Bala, "Routing in a linear lightwave network," IEEE/ACM Trans. Networking, vol. 3, pp. 459-469, Aug. 1995.

[2] I. Chlamtac, A. Ganz, and G. Karmi, "Lightpath communications: An approach to high-bandwidth optical WAN's," IEEE Trans. Commun., vol. 40, pp. 1171-1182, July 1992.

[3] R. Ramaswami and K. N. Sivarajan, "Routing and wavelength assignment in all-optical networks," IEEE/ACM Trans. Networking, vol. 3, pp. 489-500, Oct. 1995.

[4] R. A. Barry and P. A. Humblet, "Models of blocking probability in all-optical networks with and without wavelength changers," IEEE $J$. Select. Areas Commun., vol. 14, pp. 858-867, June 1996.

[5] C. Saxtoft and P. Chidgey, "Error rate degradation due to switch crosstalk in large modular switched optical networks," IEEE Photon. Technol. Lett., vol. 5, pp. 828-831, July 1993.

[6] E. L. Goldstein and L. Eskildsen, "Scaling limitations in transparent optical networks due to low-level crosstalk," IEEE Photon. Technol. Lett., vol. 7, pp. 93-94, 1995.

[7] A. R. Chraplyvy, "Limits on lightwave communications imposed by optical-fiber nonlinearities," J. Lightwave Technol., vol. 8, pp. 1548-1557, Oct. 1990.

[8] J. Gimlett, M. Iqbal, N. Cheung, A. Righetti, F. Fontana, and G. Grasso, "Observation of equivalent Rayleigh scattering mirrors in lightwave systems with optical amplifiers," IEEE Photon. Technol. Lett., vol. 2, pp. 211-213, 1990

[9] J. Gimlett and N. Cheung, "Effects of phase-to-intensity noise conversion by multiple reflections on gigabit-per-second DFB laser transmission systems," J. Lightwave Technol., vol. 7, pp. 888-895, 1989.

[10] E. Desurive, Erbium-Doped Fiber Amplifers, Principles and Applications. New York, NY: Wiley, 1994.

[11] Y. Sun and A. K. Srivastava, "Dynamic effects in optically amplified networks," in Proc. Optical Amplifiers and Their Applications (M Zervas, A. Willner, and S. Sasaki, Eds.), vol. 16, Trends of Optics and Photonics Series (TOPS), (Washington, DC), pp. 333-353, Optical Society of America, 1997.

[12] Y. Sun, A. K. Srivastava, J. L. Zyskind, J. W. Sulhoff, C. Wolf, and R. W. Tkach, "Fast power transients in WDM optical networks with cascaded EDFA's," Electron. Lett., vol. 33, pp. 313-314, 1997.

[13] Y. Sun, C. Luo, J. L. Zyskind, A. A. M. Saleh, A. K. Srivastava, and J. W. Sulhoff, "Model for gain dynamics in erbium-doped fiber amplifiers," Electron. Lett., vol. 32, pp. 1490-1491, Aug. 1996.

[14] B. Ramamurthy, Efficient Design of Wavelength-Division Multiplexing (WDM)-Based Optical Networks, Ph.D. dissertation, Dep. Comput. Sci. Univ. California, Davis, July 1998.

[15] R. A. Spanke, "Architectures for guided-wave optical space switching systems," IEEE Commun. Mag., vol. 25, pp. 42-48, 1987.

[16] J. Zhou, M. J. O'Mahony, and S. D. Walker, "Analysis of optical crosstalk effects in multi-wavelength switched networks," IEEE Photon. Technol. Lett., vol. 6, pp. 302-305, Feb. 1994.

[17] C. R. Giles and E. Desurvire, "Modeling erbium-doped fiber amplifiers," J. Lightwave Technol., vol. 9, pp. 271-283, Feb. 1991.

[18] H. Takahashi, K. Oda, and H. Toba, "Impact of crosstalk in an arrayedwaveguide multiplexer on $N \times N$ optical interconnection," J. Lightwave Technol., vol. 14, pp. 1097-1105, June 1996.

[19] G. Keiser, Optical Fiber Communications, 2nd ed. New York: McGraw-Hill, 1991.

[20] T. G. Hodgkinson, "Improved average power analysis technique for erbum-doped fiber amplifiers," IEEE Photon. Technol. Lett., vol. 4, pp. 1273-1275, 1992

[21] A. A. M. Saleh, R. M. Jopson, J. D. Evankow, and J. Aspell, "Modeling of gain in erbium-doped fiber amplifier," IEEE Photon. Technol. Lett., vol. 2, pp. 714-717, 1990

[22] H. S. Hinton, "Photonic switching using directional couplers," IEEE Commun. Mag., vol. 25, pp. 16-26, May 1987.

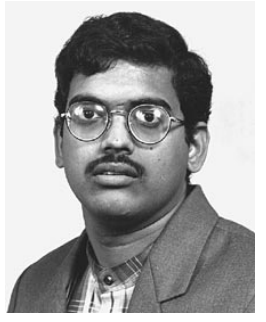

Byrav Ramamurthy (S'97-M'99) received the B.Tech. degree in computer science and engineering from Indian Institute of Technology, Madras, India, in 1993. He received the M.S. and Ph.D. degrees in computer science from University of California (UC), Davis, in 1995 and 1998, respectively.

Since August 1998, he has been an Assistant Professor in the Department of Computer Science and Engineering at the University of NebraskaLincoln (UNL), Lincoln, NE. He is the founding Co-Director of the Advanced Networking and Distributed Experimental Systems (ANDES) Laboratory at UNL.

Dr. Ramamurthy is a member of the IEEE Communications Society. $\mathrm{He}$ served as a member of the technical program committees for the IEEE GLOBECOM'99 Symposium on High Speed Networks, the IEEE International Conference on Networks (ICON'99) Conference, and the Eighth IEEE International Conference on Computer Communications and Networks (ICCCN'99). He is the Feature Editor for Theses for the Optical Network Magazine. He is a Co-Guest Editor of the upcoming Special Issue on Optical Communication Networks of the IEEE NETWORK.

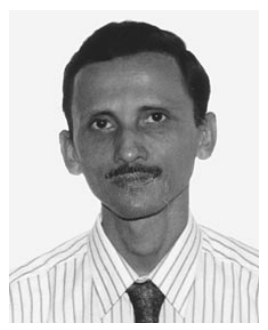

Debasish Datta received the B.Tech. degree in radiophysics and electronics from the Institute of Radiophysics and Electronics, Calcutta University, India, in 1973 and the M.Tech. and Ph.D. degrees in electronics and electrical communication engineering from the Indian Institute of Technology (IIT), Kharagpur, India, in 1976 and 1987, respectively.

From 1976 to 1978, he worked as an Assistant Executive Engineer in the Transmission R\&D Division, Indian Telephone Industries Ltd., Bangalore Since 1978, he has been engaged in research and teaching in the broad area of telecommunication systems in the Indian Institute of Technology, Kharagpur, with specific interest in the area of optical communication systems and networks. At present, he is a Professor in the Department of Electronics and Electrical Communication Engineering, and also serves as the Chairman in the G.S. Sanyal School of Telecommunication at IIT Kharagpur. During the period between 1980 and 1981 he worked as a Production Manager at Philips (India) Limited, Calcutta. During 1992-1993, he visited Stanford University, Stanford, CA, for a one-year sabbatical to carry out research in the Department of Electrical Engineering on the impact of fiber nonlinearities on coherent optical communication systems. During 1997-1998 and then during the summer of 1999, he visted University of Callifornia, Davis, to carry out research in the Department of Computer Science in the area of wavelength division multiplexed optical networks. His current research interests include optical communication systems, wavelength division multiplexed optical networks, and broad-band access networks.

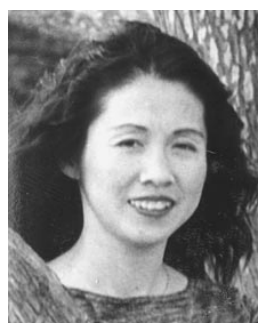

Helena Feng (S'99) received the B.S. degree in physics from Wuhan University, Wuhan, China, in 1984. She received the M.S. degree in physics from San Francisco State University, San Francisco, CA, in 1991 and the M.S. degree in applied science from University of California, Davis in 1995. Currently, she is pursuing the Ph.D. degree in the Department of Applied Science at University of California, Davis.

Her research interests are in the areas of optical device modeling and simulation of optical networks. In particular, she is interested in studying network performance using computer simulations at the physical layer as well as the network layer. She is also a Student Employee at the Lawrence Livermore National Laboratory (LLNL), Livermore, CA. Her assignments at LLNL include performing measurements of the EDFAs and BER on the National Transparent Optical Network (NTON) test bed and developing computer models and simulations of the optical fiber links to aid the deployment and expansion of the network.

Ms. Feng is a student member of IEEE COMSOC and LEOS. 


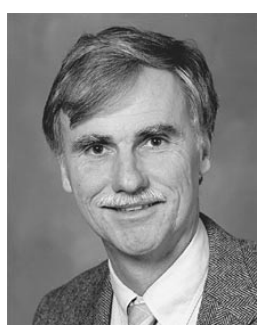

Jonathan P. Heritage (S'74-M'75-SM'89-F'90) received the $\mathrm{Ph} . \mathrm{D}$. degree from the University of California, Berkeley, in 1975.

He was a Postdoctoral Fellow of the Alexander von Humboldt Stiftung at the Physics Department of the Technical University of Munich, Germany. He was a member of staff at AT\&T Bell Laboratories, NJ, from 1976 to 1984 . In 1984, he joined the staff at the newly formed Bell Communications Research, where he became Distinguished Member of Professional Staff. In July 1991. He joined the faculty of the Department of Electrical and Computer Engineering at the University of California, Davis. He is currently Professor of Electrical Engineering and Computer Science, University of California, Davis. He has been author of more than 90 papers and six patents. He has been an active researcher in picosecond and femtosecond quantum electronics and nonlinear optics for 25 years. In 1999, he was awarded joint appointment with the Department of Applied Science, UC Davis Livermore. His present interests are in optical communications, including device and link BER modeling of WDM networks. His interests also include silicon microphotonics for freespace optical switches and femtosecond pulse shaping. His interests also range to microphotonic structures for photofield emission in vacuum and the interaction of high-field optical pulses with relativistic electrons.

Dr. Heritage is a past Associate Editor of the IEEE Journal of Quantum EleCtronics and IEEE Photonic TeChNology LetTERs. He coedited a special edition of the IEEE Journal of QuANTUM Electonics on Ultrafast Optics and Electronics. He is active in IEEE Lasers and Electro-Optics Society and is a past-elected member of the Board of Governors. He is the Co-Originator and Co-Chair of the 1992 and 1990 IEEE LEOS/COMSOC Summer Topical Meeting on Optical Multiple Access Networks. He was Technical Program Chair of the LEOS IEEE'94 Annual Meeting, Member at Large for LEOS'95 and was the General Chair for LEOS'96. He is Program Chair of the 1999 24th International Conference on Infrared and Millimeter Waves. He is a Fellow of the Optical Society of America (OSA) and the American Physical Society.

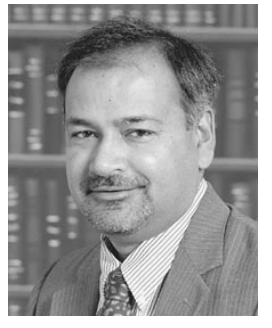

Biswanath Mukherjee (S'82-M'87) received the B.Tech. (Hons.) degree from Indian Institute of Technology (IIT), Kharagpur, India, in 1980 and the Ph.D. degree from University of Washington, Seattle in June 1987.

At the University of Washington, he held a GTE Teaching Fellowship and a General Electric Foundation Fellowship. In July 1987, he joined the University of California, Davis, where he has been Professor of Computer Science since July 1995, and Chairman of Computer Science since September 1997. He is author of the textbook Optical Communication Networks (New York: McGraw-Hill, 1997), a book which received the Association of American Publishers, Inc.'s 1997 Honorable Mention in Computer Science. His research interests include lightwave networks, network security, and wireless networks.

Dr. Mukherjee is co-winner of paper awards presented at the 1991 and the 1994 National Computer Security Conferences. He serves on the editorial boards of the IEEE/ACM TRANSACtions on NETwORKING, IEEE Network, ACM/Baltzer Wireless Information Networks (WINET), Journal of HighSpeed Networks, Photonic Network Communications, and Optical Network Magazine. He also serves as Editor-at-Large for optical networking and communications for the IEEE Communications Society. He served as the Technical Program Chair of the IEEE INFOCOM'96 Conference. 